\title{
Impact of Tissue Electromagnetic Properties on Radiation Performance of In-Body Antennas
}

\author{
Denys Nikolayev, Member, IEEE, Maxim Zhadobov, Senior Member, IEEE, and Ronan Sauleau, Fellow, IEEE
}

\begin{abstract}
In-body antennas couple strongly to surrounding biological tissues thus resulting in radiation efficiencies well below $1 \%$. Here, we quantify how the permittivity and conductivity, each individually, affect the radiation efficiency of miniature implantable and ingestible antennas. We use a generic pill-sized capsule antenna and a spherical homogeneous phantom with its electromagnetic properties covering the complete range of body tissues. In addition to the phantom surrounded by air, we study the case with a reduced phantom-background contrast (nonresonant case) that allows for decoupling of the obtained results from the phantom shape. The results demonstrate that, for a realistic capsule antenna, the effect of dielectric loading by tissue can partially compensate for the tissue losses. For instance, the gain of the antenna operating in the muscleequivalent medium is about two times $(3 \mathrm{dBi})$ higher than in the fat-equivalent one, even though the conductivity of muscle is one order of magnitude higher than the one of fat. The results suggest that, in majority of cases, in-body devices should be designed for and be placed within higher permittivity tissues with low to moderate losses.
\end{abstract}

Index Terms-biomedical telemetry, implantable, in-body, ingestible, ISM (industrial, scientific, and medical) band.

\section{INTRODUCTION}

$\mathbf{I}$ MPLANTABLE and ingestible (in-body) devices for biomedical telemetry, telemedicine, and neural interfacing provide breakthrough capabilities for both practitioners and researchers [1]-[3]. Furthermore, new applications emerge in defense, professional sports, and occupational health.

A wireless in-body device relies on antennas to interface with external systems [4]-[7]. However, the electromagnetic (EM) energy transfer between an in-body device and an external transceiver is generally inefficient. Radiofrequency (RF) transmission within about $200 \mathrm{MHz}-2 \mathrm{GHz}$ band yields the best efficiency for both data [8] and wireless power transfer [9]. The optimal frequency-in terms of the antenna radiation efficiency $\eta$-depends most notably on the depth of implantation: the closer the antenna is to the surface, the higher the optimal frequency [8], [9].

For a given depth, radiation efficiency of an in-body antenna depends on EM properties of surrounding tissues, i.e. on the

Manuscript received May 30, 2018.

This work was supported in part by the BodyCap Company, in part by the French National Center for Scientific Research and DGA through the PEPS program, and in part by Rennes Metropole through the AES program. (Corresponding author: Denys Nikolayev.)

D. Nikolayev is with the Imec / Ghent University, 9052 Gent, Belgium (e-mail: d@deniq.com)

M. Zhadobov and R. Sauleau are with the Institut d'Électronique et de Télécommunications de Rennes, UMR CNRS 6164, Université de Rennes 1, 35042 Rennes, France. dispersive complex permittivity $\hat{\varepsilon}=\varepsilon^{\prime}+j \varepsilon^{\prime \prime}$. Real part $\varepsilon^{\prime}$ loads the antenna, thus increasing its electrical size $k a \propto \eta$ (where $k$ is the wavenumber and $a$ is the antenna circumradius) [10]. Propagating wave undergoes attenuation due to losses in tissues (characterized by $\varepsilon^{\prime \prime}=\sigma / \omega$, were $\sigma$ is the conductivity and $\omega$ is the angular frequency) and scattering because of tissue heterogeneity $\hat{\varepsilon}(\mathbf{r}, \omega)$.

Several authors studied how the tissue permittivity $\varepsilon_{r}$ and conductivity $\sigma$ affect the radiation performance of in-body antennas. Skrivervik and co-workers [11], [12] characterized losses of ideal electric, magnetic, and Huygens sources in a multilayer spherical phantom using spherical wave decomposition. The authors quantified the role of the antenna type and encapsulation aiming at minimizing the losses by lowering the near-field coupling. Chrissoulidis and Laheurte [13] employed a similar spherical body model to study the radiation of a dipole using a dyadic Green's function approach. The results characterize the near field of ideal electric and magnetic antennas. Xu et al. [14], [15] evaluated numerically the specific absorption rate (SAR) of an ingestible helical antenna in gastrointestinal tract tissues. In our recent study [8], we derived optimal radiation conditions for idealized sources in realistic phantoms. However, to the best of our knowledge, the quantitative effect of tissue EM properties on radiation performance of capsule in-body antennas remains unexplored.

In this letter, we study the impact of the tissue permittivity $\varepsilon_{r}$ and conductivity $\sigma$ on the gain $G$ of a pill-sized in-body capsule antenna. We start by formulating the problem for a conformal-microstrip antenna in canonical phantoms. Next, we study the effect of tissue EM properties on radiation performances. We quantify the effect of $\left\{\varepsilon_{r}, \sigma\right\}$ on the antenna radiation efficiency losses. Finally, we experimentally validate the numerical results.

\section{Problem Formulation}

\section{A. Antenna Design}

We used a representative microstrip design conforming to an inner surface of a 0.5 -mm-thick $17 \mathrm{~mm} \times \varnothing 7 \mathrm{~mm}$ capsule (Fig. 1) [16]. The operating band is $434 \mathrm{MHz}$ ISM (Industrial, Scientific, and Medical) [17]. The antenna is loaded with ceramic shell $\left(\mathrm{Al}_{2} \mathrm{O}_{3}, \varepsilon_{r} \approx 10\right)$ and pure water inner filling $\left(\varepsilon_{r} \approx 78\right)$. This approach results in stable $50-\Omega$ matching (i.e. $\left|S_{11}\right|<-10 \mathrm{~dB}$ ) when operating in the majority of biological tissues for both implantable and ingestible applications. This makes this design an appropriate candidate to study the effect 
of variations of tissue EM properties on the antenna radiation performance.

\section{B. Spherical Phantom}

Studying the effect of tissue EM properties $\left\{\varepsilon_{r}, \sigma\right\}$ on the antenna radiation requires decorrelation of the results from the phantom geometry. Therefore, any complex shapes and heterogeneities will introduce shape-related disturbances to the results. Spherical symmetry of the phantom allows for characterization of radiation performance under well-controlled isotropic conditions. It conserves the intrinsic radiation pattern of an in-body antenna and can be reproduced in measurements [16]. The diameter of the sphere has to reflect the potential in-body application scenarios (i.e. the mean implantation depth) and, for the human-ingestible one, it is in $\varnothing 60-200 \mathrm{~mm}$ range [18]. We chose $\varnothing 100$-mm (Fig. 1) as it ensures at least half-wavelength propagation for $f_{0} \geq 434 \mathrm{MHz}$ and $\varepsilon_{r} \geq 50$ (the permittivity range $\varepsilon_{r} \in[50 . .80]$ covers all water-based tissues [19]). Already at this diameter, the radiation efficiency of in-body antennas usually drops to $\eta<1 \%$ [18]. We define the EM properties of the specified phantom to be within the following range: $\varepsilon_{r} \in[10 . .80]$ and $\sigma \in[0 . .2 .4] \mathrm{S} \cdot \mathrm{m}^{-1}$ [19].

First, we study the radiation from the phantom in free space $\left\{\varepsilon_{0}, 0\right\}$. Because of the high contrast boundary between the lossless phantom and free space, the sphere may act as a dielectric resonator. This resonance affects the gain and thus introduces an additional geometry-related disturbance. We decorrelated the results by matching the permittivity of the environment to the one of the phantom, hence mitigating the high-contrast boundary between the phantom and free space. This makes the phantom nonresonant and enables an accurate estimate of how the radiation performance of the antenna depends on the EM properties of surrounding tissues.

To make the results independent of the antenna design, we normalize the computed gain to its maximum value over $\left\{\varepsilon_{r}, \sigma\right\}$ as $G_{\text {norm, } \mathrm{dBi}}=G_{\mathrm{dBi}}\left(\varepsilon_{r}, \sigma\right)-\max \left(G_{\mathrm{dBi}}\right)$. In this way, $G_{\text {norm }}$ quantifies the response of the radiation efficiency $\eta$ to the variations of $\left\{\varepsilon_{r}, \sigma\right\}$. Considering the effect of dielectric loading on the antenna radiation, the highest permittivity $\varepsilon_{r}=80$ and the lowest conductivity $\sigma=0$ considered in this study result in $\max \left(G_{\mathrm{dBi}}\right)$. These EM properties are close to these of pure water at $434 \mathrm{MHz}$.

As the directivity $D$ is almost constant for the studied range of EM properties ( $D \approx 2 \mathrm{dBi} \forall\left\{\varepsilon_{r}, \sigma\right\}$ ), the normalized gain $G_{\text {norm, dBi values can be considered equivalent }}$ to the normalized radiation efficiency $\eta_{\text {norm, } \mathrm{dB}}\left(\varepsilon_{r}, \sigma\right)=$ $\eta_{\mathrm{dB}}\left(\varepsilon_{r}, \sigma\right)-\max \left(\eta_{\mathrm{dB}}\right)$ as $G=\eta D[20]$.

\section{NUMERICAL ANALYSIS}

\section{A. Numerical Model}

We used the frequency domain solver of CST Microwave Studio 2017 [21] (finite element method) as the most suitable for resolving the geometry of a low-profile conformal antenna. Adaptive mesh refinement used two convergence criteria: i) $\delta\left|S_{11}\right|<1 \%$ for three consecutive passes and ii) $\delta \max (G)<2 \%$ for two consecutive passes. The open boundaries were modeled as the perfectly matched layers (PMLs); the distance from the phantom to PML was $\lambda_{0} / 2$.

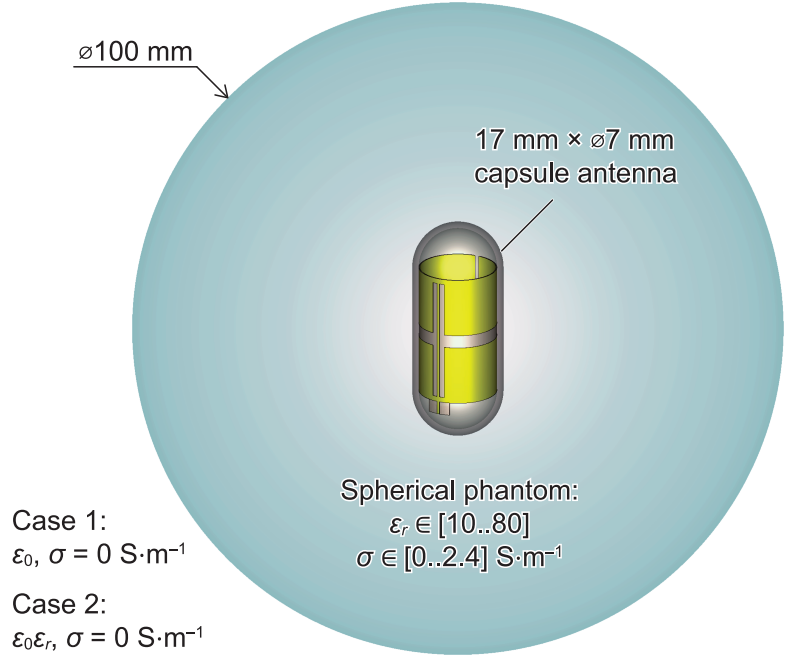

Fig. 1. Problem formulation (not to scale): the capsule antenna centered inside a canonical $\varnothing 100-\mathrm{mm}$ spherical phantom covering the complete range of biological tissue EM properties at $434 \mathrm{MHz}$. Two cases were studied: 1) a phantom surrounded by free space with $\varepsilon_{0}$, and 2) a nonresonant phantom surrounded by a medium with $\varepsilon_{r}$.

\section{B. Radiation from Phantom Surrounded by Air}

Fig. 2a shows the effect of $\left\{\varepsilon_{r}, \sigma\right\}$ on the antenna radiation. The gain is normalized by its global maximum at about $\{45,0\}$ where the phantom is resonant (radius $\approx \lambda / 2$ ). The local maximum is at $\{80,0\}$ and the global minimum is at $\{10,2.4\}$ - the boundary values within the studied range. The max-min ratio reaches $33.3 \mathrm{dBi}$.

Clearly, the tissues load the antenna that increases its electrical size as $k a \propto \sqrt{\varepsilon_{r}}$. The achievable radiation efficiency $\eta_{\max }$ is proportional to $k a$ for any electrically-small antenna [10]. This effect allows for partial compensation of the losses induced by the conductivity. For instance, the gain of the antenna operating in the phantom with stomach-equivalent EM properties is about two times higher than in the fat-equivalent one (Fig. 2a). This is despite the fact that the conductivity of stomach being one order of magnitude higher than the one of fat. The beneficial effect remains until conductivity reaches $\sigma \lesssim 1 \mathrm{~S} \cdot \mathrm{m}^{-1}$. For instance, the losses in small intestine are higher than in fat.

To quantify the effect of dielectric loading by tissue with respect to the reference gain at $\{80,0\}$, let us now consider a nonresonant phantom.

\section{Radiation from Nonresonant Phantom}

Matching the permittivity of the phantom to the one of the outer space mitigates the resonance at $\{45,0\}$, and the $\max (G)$ moves to the reference point $\{80,0\}$ (Fig. 2b). Even though, at the considered frequencies, the conductivity of fat and pure water are of the same order, the eightfold difference in $\varepsilon_{r}$ results in the relative radiation efficiency loss of $-15 \mathrm{~dB}$. For the muscle tissue, this value is $-11 \mathrm{~dB}$ that is comparable to the loss from colon and stomach tissues. However, increased conductivity of the small intestine gives the relative loss of about $-17 \mathrm{dBi}$. 
TABLE I

Polynomial CoefFicients of EQ. 1

\begin{tabular}{ccccccccc}
$p_{00}$ & $p_{10}$ & $p_{01}$ & $p_{20}$ & $p_{11}$ & $p_{02}$ & $p_{21}$ & $p_{12}$ & $p_{03}$ \\
\hline-25.89 & 3.683 & -4.968 & -0.57 & -0.8826 & 0.8397 & 0.4229 & 0.1169 & -0.2408
\end{tabular}

For the studied range of $\left\{\varepsilon_{r}, \sigma\right\}$, one can accurately predict the gain loss $G_{\text {norm }}(\mathrm{dBi})$ with respect to the reference point $\{80,0\}$ for a canonical $\varnothing 100-\mathrm{mm}$ spherical phantom (adjusted coefficient of determination $\left.\bar{R}^{2}=0.9962\right)$ :

$$
\begin{gathered}
G_{\text {norm }}(\mathrm{dBi})=p_{00}+p_{10} \varepsilon_{r}+p_{01} \sigma+p_{20} \varepsilon_{r}^{2} \\
+p_{11} \varepsilon_{r} \sigma+p_{02} \sigma^{2}+p_{12} \varepsilon_{r} \sigma^{2}+p_{03} \sigma^{3}
\end{gathered}
$$

where the $p_{i j}, i=0,1,2 ; j=0,1,2,3$ are the polynomial coefficients (the values are given in Table I).

\section{Effect of Depth}

Up to this point, we have not considered how the depth of an in-body antenna affects its radiation performance. The maximum achievable radiation efficiency at a given frequency is limited by 1) the attenuation in tissue and 2) the behavior of radiated wave at high-contrast boundaries such as tissue-air (reflection, surface wave effects, etc.) [8]. The attenuation is proportional to the depth of the antenna in tissue. To quantify its effect on $G_{\text {norm }}$ (1), we studied the antenna radiation from a muscle-equivalent nonresonant phantom ranging in diameter from zero (lossless case) to $\varnothing 200 \mathrm{~mm}$. As Eq. (1) is valid for the $\varnothing 100-\mathrm{mm}$ phantom, we use it as a reference point for the normalization of the obtained results. Fig. 3 shows the gain difference $\Delta G$ in phantoms with various diameters (i.e. different distances from the antenna to the tissue boundary). The results can be linearly approximated (adjusted coefficient of determination $\bar{R}^{2}=0.999$ ) as

$$
\Delta G=-0.9084 D+8.983,
$$

where $D$ is the phantom diameter $(\mathrm{cm})$.

Eq. (1) can be adjusted using (2) for a given depth as $G_{\text {adj }}=$ $G_{\text {norm }}+\Delta G(\mathrm{dBi})$. The phantom diameter can be translated to the implantation depth as $D / 2-8.5 \mathrm{~mm}$ if the capsule is perpendicular to the skin and $D / 2-3.5 \mathrm{~mm}$ if parallel.

\section{Measurements}

The antenna prototype was manufactured using laser ablation on a $50 \mu \mathrm{m}$ Rogers ULTRALAM 3850HT substrate, then assembled (Fig. 4) following the procedure reported in [16]. We characterized the prototype in two media: 1) in a muscle-equivalent phantom and 2$)$ in pure water $\left(\left\{\varepsilon_{r}, \sigma\right\}\right.$ close to the reference point $\{80,0\})$. The muscle-equivalent EM properties were achieved using a water-sugar-salt formulation (see details in [16]). Sucrose $\left(\mathrm{C}_{12} \mathrm{H}_{22} \mathrm{O}_{11}, \geq 99.5 \%\right)$ reduced the permittivity $\varepsilon_{r}$ of pure water, and sodium chloride $(\mathrm{NaCl}$, $\geq 99.5 \%$ ) increased the conductivity $\sigma$. To characterize the EM properties of the phantom, we used the SPEAG DAK kit with a DAK-12 probe. Fig. 5 shows the measured EM properties of the phantom compared to the theoretical ones [19].

First, we characterized the antenna in terms of its reflection coefficient $\left|S_{11}\right|$. This allows for the preliminary validation

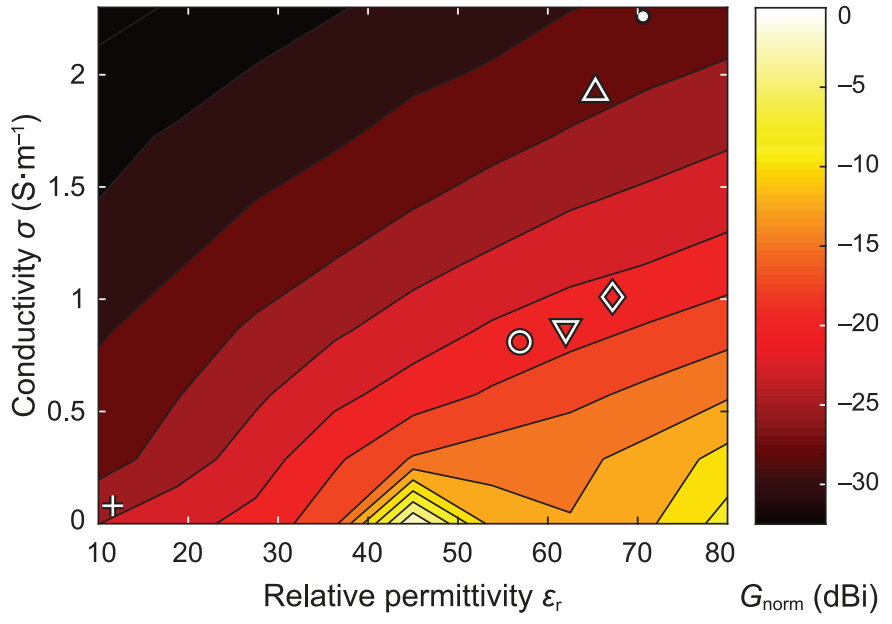

(a)

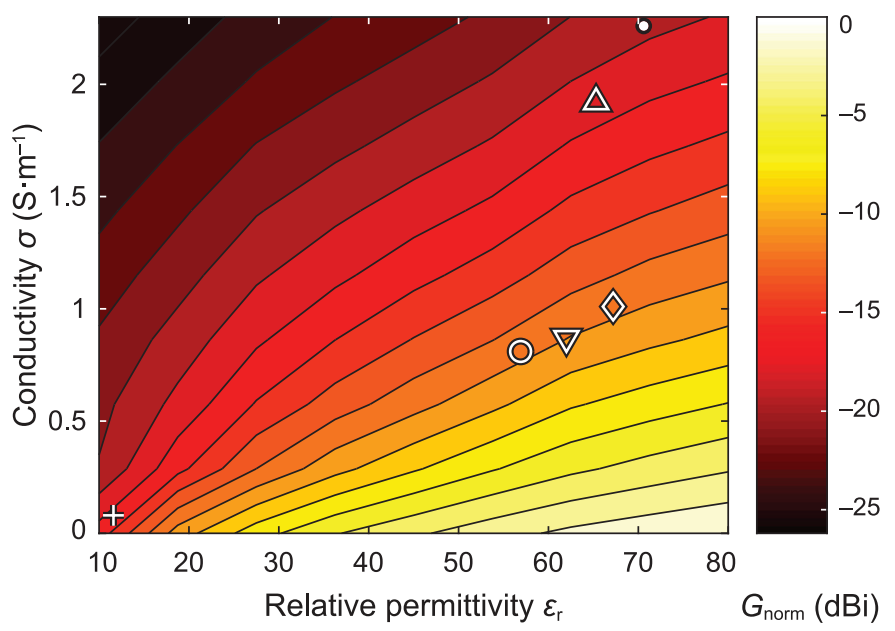

(b)
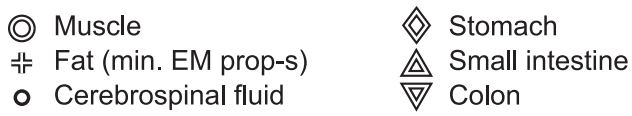

Fig. 2. Normalized gain in a $\varnothing 100$-mm spherical phantom covering the complete range of biological tissue EM properties. (a) Phantom in free space. (b) Nonresonant phantom (environment $\varepsilon_{r}=$ phantom $\varepsilon_{r}$ ).

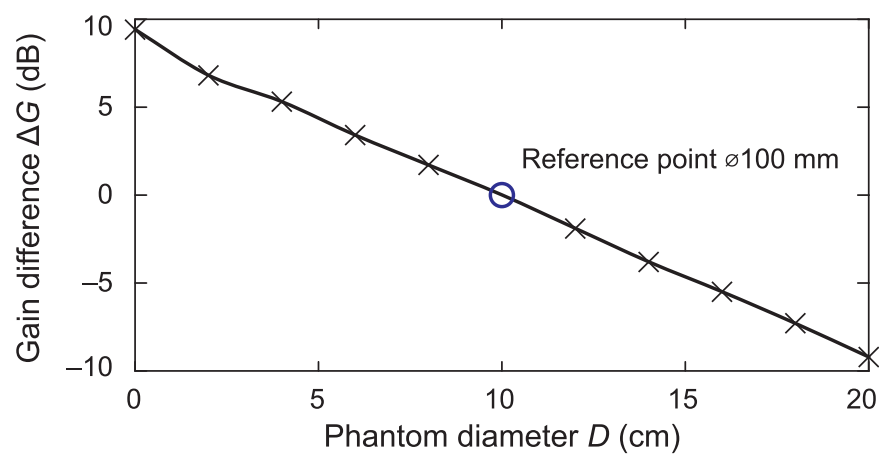

Fig. 3. Gain difference $\Delta G$ of the antenna operating within the muscleequivalent phantom of various sizes.

of the numerical model as well as for the calculation of the gain $G$ from the measured total gain $G_{\text {tot }}=\left(1-|\Gamma|^{2}\right) G$, where $\Gamma$ is the voltage reflection coefficient of the antenna 
[20]. Fig. 6 shows $\left|S_{11}\right|$ of the antenna measured in muscleequivalent phantom and pure water.

To contain the liquids for radiation characterization, we used a $\varnothing 100$-mm spherical glass jar (wall thickness $\approx 2 \pm 1 \mathrm{~mm}$ ) with the antenna centered inside (AuT, Fig. 4a). A foam frame (Rohacell IG, $\varepsilon_{r}=1.05, \tan \delta=0.0017$ ) allows for the accurate positioning of the AuT in an anechoic chamber.

Considering the electrical and physical size of the capsule antenna as well as its radiation efficiency in phantoms $\eta \sim$ $0.1 \%$, one cannot use conventional approaches to accurately measure the radiation [22]. In this study, we combined three techniques (Fig. 4b): 1) illuminating the AuT in a fullyanechoic chamber (direct illumination in far-field); 2) feeding the AuT using an optical fiber connecting the base unit (enprobe LFA-3, outside of the anechoic chamber) with an electro-optical converter located at the bottom of the phantom; and 3 ) inside of the phantom, decoupling the coaxial feed from the liquids by a layer of air [23]. For the latter, we used a $\varnothing 10$-mm polyamide tube sealed with silicone. To estimate the gain, we used the gain substitution technique with a reference antenna of known gain (ETS-Lindgren 3164-06).

In the muscle-equivalent phantom, the measured maximum gain is $-19.6 \mathrm{dBi}$. Considering the electrical size and radiation efficiency of the antenna, this result agrees fairly well with the model that predicts the gain of $-22.4 \mathrm{dBi}$. The measured gain in pure water (considering the mismatch loss of $1 \mathrm{~dB}$, Fig. 6) is $-15.3 \mathrm{dBi}$; the model predicts the gain of $-11.3 \mathrm{~dB}$. The measurement error can be attributed, in particular, to the antenna manufacturing methods (laser ablation generally has lower precision than photolithography), high sensitivity of the antenna placement inside of the phantom, and irregular thickness of the phantom glass wall $(\approx 2 \pm 1 \mathrm{~mm})$.

\section{Discussion And Conclusion}

We analyzed the impact of the permittivity and conductivity of biological tissues on the gain of a representative in-body conformal-microstrip antenna. We quantified the relative gain loss when radiating from media covering the full range of EM properties of tissues. Even though the attenuation constant (for any transverse-electromagnetic wave) is $\alpha \propto \sigma \sqrt{\varepsilon^{\prime}}$, the results demonstrate that the effect of dielectric loading can compensate for the losses in adjacent tissues if conductivity remains below $\sigma \lesssim 1 \mathrm{~S} \cdot \mathrm{m}^{-1}$. For instance, the gain of the antenna operating in the muscle-equivalent medium is about two times $(3 \mathrm{dBi})$ higher than in the fat-equivalent one even though the conductivity of muscle being one order of magnitude higher than the one of fat.

This effect has implications for both engineers and practitioners: in majority of cases, in-body devices should be designed for and be placed within a higher permittivity tissues. Obviously, the reflection coefficient of the antenna must remain at an acceptable level (e.g. $\mid S_{11}<-10 \mathrm{~dB}$ ) to avoid mismatch loss. Dielectric loading by capsule materials can be used as well to improve the radiation efficiency. This approach was demonstrated in [24] with an added benefit of increased robustness.

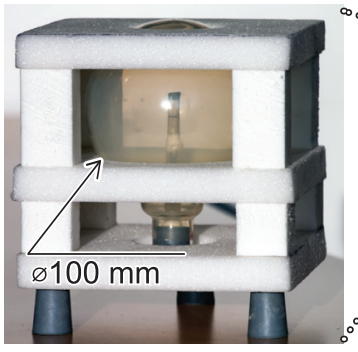

(a)

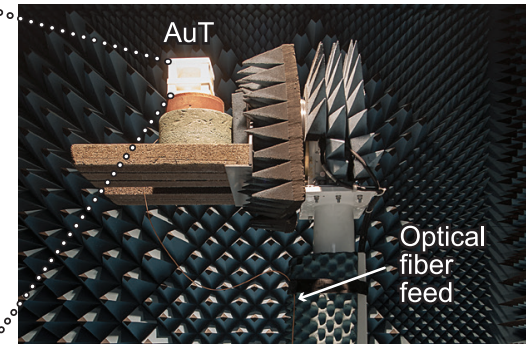

(b)
Fig. 4. Experimental validation of the results. (a) Capsule antenna mockup centered inside of a $\varnothing 100-\mathrm{mm}$ spherical container. (b) Far-field characterization setup inside of an anechoic chamber.

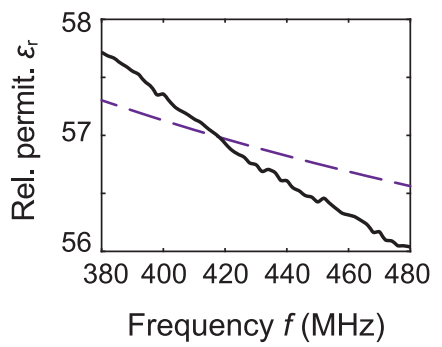

(a)

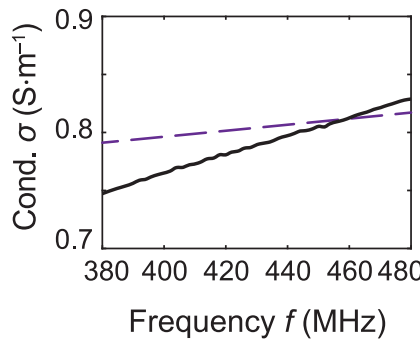

(b)
Fig. 5. Measured (-) and theoretical (- - ) EM properties of the muscleequivalent liquid phantom used for the radiation measurements. (a) Relative permittivity $\varepsilon_{r}$. (b) Conductivity $\sigma$.

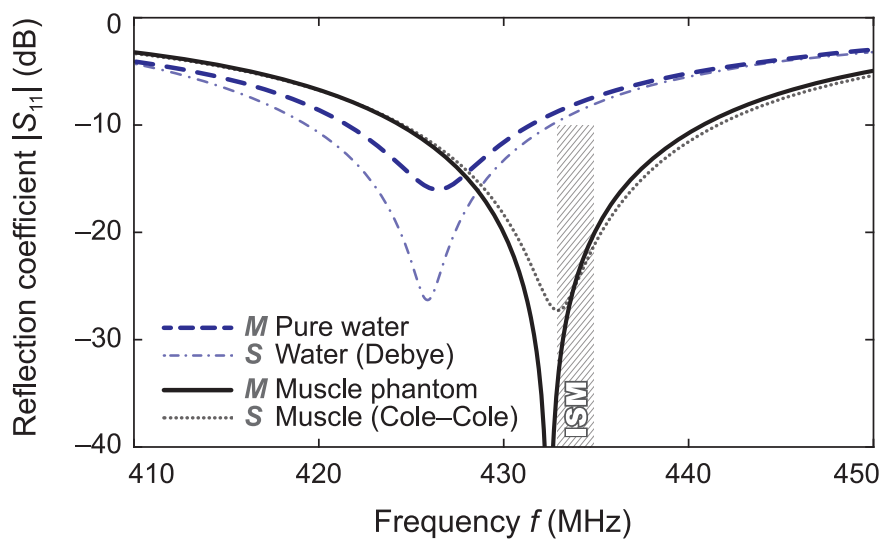

Fig. 6. Measured and computed reflection coefficients $\left|S_{11}\right|$ of the capsule antenna ( $\boldsymbol{M}$ is the measurement and $\boldsymbol{S}$ is the simulation).

The findings of this study apply to the MedRadio frequency bands too [25] as both the tissue EM properties [19] and antenna performance [16] vary insignificantly within the 401$457 \mathrm{MHz}$ range. However, at $2.45 \mathrm{GHz}$ the effect of $\left\{\varepsilon_{r}, \sigma\right\}$ should be studied separately, as pill-sized in-body antennas are no longer electrically small $(k a>0.5)$ and the tissue EM properties vary significantly from the ones at $434 \mathrm{MHz}$ [19]. In addition, this study considered a ceramic-loaded microstrip antenna to evaluate the normalized gain loss. Other antenna types (e.g. loop antennas) may have different nearfield distribution thus affecting the electric field dissipation in tissues adjacent to the antenna. 


\section{REFERENCES}

[1] A. Kiourti and K. S. Nikita, "A review of in-body biotelemetry devices: implantables, ingestibles, and injectables," IEEE Trans. Biomed. Eng., vol. 64, no. 7, pp. 1422-1430, Jul. 2017.

[2] E. Katz, Implantable Bioelectronics. Weinheim, Germany: Wiley-VCH, 2014.

[3] R. Bashirullah, "Wireless implants," IEEE Microw. Mag., vol. 11, no. 7, pp. S14-S23, Dec. 2010.

[4] Z. Bao, Y. X. Guo, and R. Mittra, "An ultrawideband conformal capsule antenna with stable impedance matching," IEEE Trans. Antennas Propag., vol. 65, no. 10, pp. 5086-5094, Oct. 2017.

[5] J. Faerber et al., "In vivo characterization of a wireless telemetry module for a capsule endoscopy system utilizing a conformal antenna," IEEE Trans. Biomed. Circuits Syst., vol. 12, no. 1, pp. 95-105, Feb. 2017.

[6] M. K. Magill, G. A. Conway, and W. G. Scanlon, "Tissue-independent implantable antenna for in-body communications at $2.36-2.5 \mathrm{GHz}$,' IEEE Trans. Antennas Propag., vol. 65, no. 9, pp. 4406-4417, Sep. 2017.

[7] S. Bakogianni and S. Koulouridis, "On the design of miniature MedRadio implantable antennas," IEEE Trans. Antennas Propag., vol. 65, no. 7, pp. 3447-3455, Jul. 2017.

[8] D. Nikolayev, M. Zhadobov, P. Karban, and R. Sauleau, "Electromagnetic radiation efficiency of body-implanted devices," Phys. Rev. Appl., vol. 9, no. 2, pp. 024033, Feb. 2018.

[9] A. S. Y. Poon, S. O’Driscoll, and T. H. Meng, "Optimal frequency for wireless power transmission into dispersive tissue," IEEE Trans. Antennas Propag., vol. 58, no. 5, pp. 1739-1750, May 2010.

[10] A. Karlsson, "Physical limitations of antennas in a lossy medium," IEEE Trans. Antennas Propag., vol. 52, no. 8, pp. 2027-2033, Aug. 2004.

[11] A. K. Skrivervik, "Implantable antennas: The challenge of efficiency," in Proc. $7^{\text {th }}$ Eur. Conf. on Antennas and Propagation (EuCAP 2013), Gothenburg, Sweden, 2013, pp. 3627-3631.

[12] A. K. Skrivervik, M. Bosiljevac and Z. Sipus, "Design considerations for implantable and wearable antennas," in Proc. $13^{\text {th }}$ Int. Conf. on Advanced Technologies, Systems and Services in Telecommunications (TELSIKS 2017), Nis, Serbia, 2017, pp. 83-86.

[13] D. P. Chrissoulidis and J. M. Laheurte, "Radiation from an encapsulated hertz dipole implanted in a human torso model," IEEE Trans. Antennas Propag., vol. 64, no. 12, pp. 4984-4992, Dec. 2016.

[24] D. Nikolayev, M. Zhadobov, and R. Sauleau, "An efficient dual-band conformal antenna with stable $50-\Omega$ matching for versatile in-body applications," IEEE Trans. Antennas Propag., under review
[14] L. Xu, M. Q.-H. Meng, H. Ren, and Y. Chan, "Radiation characteristics of ingestible wireless devices in human intestine following radio frequency exposure at 430, 800, 1200, and $2400 \mathrm{MHz}, "$ IEEE Trans. Antennas Propag., vol. 57, no. 8, pp. 2418-2428, Aug. 2009.

[15] L. Xu, M. Q.-H. Meng, and Y. Chan, "Effects of dielectric parameters of human body on radiation characteristics of ingestible wireless device at operating frequency of $430 \mathrm{MHz}$," IEEE Trans. Biomed. Eng., vol. 56, no. 8, pp. 2083-2094, Aug. 2009.

[16] D. Nikolayev, M. Zhadobov, L. Le Coq, P. Karban, and R. Sauleau, "Robust ultra-miniature capsule antenna for ingestible and implantable applications," IEEE Trans. Antennas Propag., vol. 65, no. 11, pp. 61076119, Nov. 2017

[17] International Telecommunication Union (ITU). ITU Radio Regulations (article 5), footnotes 5.138, 5.150, and 5.280. Accessed: Feb. 20, 2018. [Online]. Available: https://www.itu.int/net/ITU-R/terrestrial/ faq/index.html\#g013

[18] D. Nikolayev, M. Zhadobov, R. Sauleau, and P. Karban, "Antennas for ingestible capsule telemetry," in Advances in Body-Centric Wireless Communication: Applications and State-of-the-Art, London, UK: IET, 2016, pp. 143-186.

[19] S. Gabriel, R. W. Lau, and C. Gabriel, "The dielectric properties of biological tissues: II. Measurements in the frequency range $10 \mathrm{~Hz}$ to 20 GHz," Phys. Med. Biol., vol. 41, pp. 2251-2269, Nov. 1996.

[20] C. A. Balanis, Antenna Theory: Analysis and Design, $4^{\text {th }}$ ed. Hoboken, NJ: John Wiley \& Sons, 2016.

[21] Computer Simulation Technology AG. CST Microwave Studio. Accessed: Feb. 20, 2018. [Online]. Available: http://www.cst.com

[22] L. Huitema, C. Delaveaud, and R. D'Errico, "Impedance and radiation measurement methodology for ultra miniature antennas," IEEE Trans. Antennas Propag., vol. 62, no. 7, pp. 3463-3473, Jul. 2014.

[23] F. Merli, L. Bolomey, J. Zurcher, G. Corradini, E. Meurville, and A. K. Skrivervik, "Design, realization and measurements of a miniature antenna for implantable wireless communication systems," IEEE Trans. Antennas Propag., vol. 59, no. 10, pp. 3544-3555, Oct. 2011.

[25] Federal Communications Commission (FCC). Medical Device Radiocommunications Service (MedRadio). Accessed: Feb. 20, 2018. [Online]. Available: https://www.fcc.gov/medical-device-radiocommunicationsservice-medradio 